\title{
Une plateforme pour donner son avis
}

\section{Kerstin Schutz}

FMH, division Tarifs et conventions pour la médecine ambulatoire en Suisse

Lors de la procédure de consultation de la FMH, tous les membres de la FMH auront la possibilité d'évaluer les propositions de révision, de donner leur avis et de proposer des modifications.

\section{INPO 'TARIFAIRE IMPOR'TAN'TE}

\section{De quoi s'agit-il}

La révision de la structure tarifaire ambulatoire avance bien - les premières équipes spécialisées ont désormais pu achever leurs travaux ou sont en phase de finalisation. En parallèle, la traduction en français bat son plein.

Une fois traduits, les différents chapitres seront publiés au fur et à mesure dans le navigateur tarifaire.

La consultation porte sur la nomenclature (français/ allemand) du tarif révisé, à savoir:

- Dénomination des chapitres

- Interprétation des chapitres

- Dénomination des positions tarifaires

- Interprétation médicale des positions tarifaires

- Minutages

- Règles techniques

Correspondance: FMH / division Tarifs et conventions pour la médecine ambulatoire en Suisse

Frohburgstrasse 15

CH-4600 Olten

Tél. 0313591230

Fax 0313591238

tarife.ambulant[at]fmh.ch

\section{Comment}

Un article sera publié dans le Bulletin des médecins suisses le 6 janvier 2016 pour annoncer l'ouverture officielle de la consultation. Dès que celle-ci sera ouverte et que tous les chapitres auront été publiés (mi-janvier 2016), les membres de la FMH recevront toutes les informations nécessaires par courrier électronique.
Les membres pourront donner leur avis sur la nomenclature à l'aide d'un formulaire de feed-back en ligne. Les remarques devront être les plus précises possible (titre, interprétation, minutage, etc.), avec motivation et dans l'idéal, une proposition alternative, pour que nous puissions les examiner en détail.

La FMH réunira tous les retours reçus et les transmettra aux équipes spécialisées dès la mi-février pour qu'elles puissent les analyser.

\section{Quand}

A partir de la mi-décembre 2015, les premiers chapitres devraient être mis à disposition dans le navigateur tarifaire en ligne.

La consultation officielle de tous les chapitres durera 4 semaines, entre mi-janvier et mi-février 2016.

Vous trouverez toutes les dernières informations à ce sujet sur le site Internet de la FMH www.fmh.ch/fr/ tarifs_ambulatoires.html ou auprès de la délégation tarifaire de votre société médicale.

Si vous souhaitez être informé personnellement dès qu'un nouveau chapitre est disponible pour la consultation, vous pouvez vous inscrire à notre lettre d'information par courriel à tarife.ambulant@fmh.ch Des informations détaillées concernant la procédure de consultation de la FMH ont également été données lors de la séance d'information du 4 novembre 2015 sur la révision de la structure tarifaire ambulatoire. Vous trouverez toutes les présentations Powerpoint dans la rubrique «Téléchargements» du portail des membres myFMH. 